\title{
Therapeutic Hypothesis Testing With Rodent Brain Tumor Models
}

\author{
Derek A. Wainwright ${ }^{1,2,3}$ - Craig M. Horbinski ${ }^{1,4}$ - Rintaro Hashizume ${ }^{1,5}$ • \\ C. David James ${ }^{1,5}$ (1)
}

Published online: 20 March 2017

(C) The American Society for Experimental NeuroTherapeutics, Inc. 2017

\begin{abstract}
The development and application of rodent models for preclinical testing of novel therapeutics and approaches for treating brain tumors has been a mainstay of neuro-oncology preclinical research for decades, and is likely to remain so into the foreseeable future. These models serve as an important point of entry for analyzing the potential efficacy of experimental therapies that are being considered for clinical trial evaluation. Although rodent brain tumor models have seen substantial change, particularly since the introduction of genetically engineered mouse models, certain principles associated with the use of these models for therapeutic testing are enduring, and form the basis for this review. Here we discuss the most common rodent brain tumor models while directing specific attention to their usefulness in preclinical evaluation of experimental therapies. These models include genetically engineered mice that spontaneously or inducibly develop brain tumors; syngeneic rodent models in which cultured tumor cells are engrafted into the same strain of rodent from which they were derived; and patient-derived xenograft models in which
\end{abstract}

C. David James

charles.james@northwestern.edu

1 Department of Neurological Surgery, Northwestern University Feinberg School of Medicine, Chicago, IL, USA

2 Department of Microbiology and Immunology, Northwestern University Feinberg School of Medicine, Chicago, IL, USA

3 Department of Medicine-Hematology/Oncology, Northwestern University Feinberg School of Medicine, Chicago, IL, USA

4 Department of Pathology, Northwestern University Feinberg School of Medicine, Chicago, IL, USA

5 Department of Biochemistry and Molecular Genetics, Northwestern University Feinberg School of Medicine, Chicago, IL, USA human tumor cells are engrafted in immunocompromised rodents. The emphasis of this review is directed to the latter.

Keywords Xenograft · Syngeneic · Immunocompetent vs. immunocompromised $\cdot$ Spontaneous vs. engraftment

\section{Genetically Engineered Mouse Models: Utility for Therapeutic Testing (?)}

Genetically engineered mouse (GEM) models have been a tool of cancer research for over a quarter of a century. Early versions were based on the constitutional inactivation of tumor suppressor genes and/or introduction of activated oncogenes into the germline, such that the progeny of genetically engineered mice would harbor genetic modifications favoring tumor development. Initial models were not developed for modeling specific types of cancer, but rather were created to determine whether specific germline alterations caused any type of cancer development [1]. In some cases the oncogenic transgenes used to promote tumor formation were not necessarily relevant to the cancer that was produced. For example, the glioma model described by Ding et al. [2] is based on the expression of a mutant Ras ( $\mathrm{V}^{12} \mathrm{Ha}$-ras $)$ transgene. Despite the rarity of Ras mutations in glial tumors, this particular GEM has seen widespread use in brain tumor research, in large part because of its reproducible and consistent tumor development. Tumors that develop present with histologic and molecular characteristics consistent with those found in patient glioblastoma multiforme (GBM), including mutation of TP53 and suppression of PTEN and CDKN2A expression, the latter of which encodes the p16 tumor suppressor. A drawback this model, and demonstrated by other GEM models, is the frequent presentation of multifocal tumor development, which is not typical of GBM in patients. 
GEM model sophistication increased rapidly during the 1990s and early 2000s, culminating with contemporary GEM possessing inducible tumor suppressor gene knockouts, oncogene knock-ins, and improved cell type-specificity control over genetic alteration induction [3]. A prime example of a contemporary GEM model is based on glial fibrillary acidic protein-associated conditional inactivation of the NF1 tumor suppressor gene in mice that are constitutionally deficient in TP53 [4]. Ras pathway activation, either by deregulated upstream receptor tyrosine kinase signaling, Ras mutation, or NF1 tumor suppressor inactivation, has been popular in GEM modeling of glial tumors. However, and in contrast to models based on the expression of mutant Ras, NF1-inactivating mutations occur frequently in malignant gliomas from patients. Humans with mutated NF1 have an increased risk of developing astrocytoma, and tumors with combined NF1- and TP53inactivating mutations frequently manifest as GBM [5]. GEM allowing for temporal, cell-type specific inactivation of $N F 1$, in the context of a p53-null background, display high penetrance for NF1 gene inactivation causing tumor formation, with tumors showing many of the hallmark features of human GBM. A derivative of this model, involving the inclusion of constitutional PTEN haploinsufficiency, increases tumor formation to $100 \%$ when NF1 is inactivated, and decreases tumor latency [6].

The GEM models have addressed and continue to address needs associated with significant shortcomings of the engraftment models. They enable the analysis of events associated with early tumor development, provide opportunity to study tumor evolution, and are not dependent on an invasive procedure, the intracranial injection of tumor cells, that disrupts the blood-brain barrier, thereby altering the tumor microenvironment. GEM models also are able to address potential brain tumor cell of origin identity. Notably, the immunocompetent status of GEM is compatible with testing immunotherapies [7].

A weakness of GEM models is that they do not, in general, compare favorably with engraftment models for therapeutic testing capacity. Reasons for this include the extensive resources, time, and costs associated GEM genotyping, breeding, and colony maintenance; asynchronous tumor development in age-matched mice of the same strain; and the infrequent inclusion of a reporter transgene that can be used for monitoring intracranial tumor development and response to therapy [8].

\section{Syngeneic, Immunocompetent Mouse Tumor Engraftment Models}

The use of rodent brain tumor cell lines, developed as a consequence of animal treatment with chemical mutagens, generally nitrosoureas, has a long history in neuro-oncology research. Some of the most commonly utilized tumor cell line-host combinations include 9 L, F98, and RG2 cells in Fisher rats, CNS1 cells in Lewis rats, GL261 and CT-2A cells in C57BL6 mice,
SMA-560 cells in VM/Dk mice, and 4C8 cells in B6D2F1 mice $[9,10]$. A literature survey indicates that GL261-C57BL6 is the most extensively used model, and, in general, mouse models have been favored, likely because of the economy of purchasing and housing mice versus rats. Although in recent years the neurooncology research community has directed more attention to the use of patient-derived xenograft models for therapeutic testing, the syngeneic, immunocompetent rodent models continue to serve a critically important role in brain tumor research, with much of the current usage directed to preclinical testing of therapies that stimulate an adaptive immune response against tumor, such as antibodies against programmed death 1, programmed death ligand 1, cytotoxic T-lymphocyte-associated protein 4, 4$1 \mathrm{BB}$, and/or OX-40, and inhibitors of specific enzymatic activities such as indoleamine 2,3-dioxygenase [11-13]. Deficiencies of the syngeneic engraftment models, and in contrast to GEM model tumors, include the relative cellular homogeneity of the intracranial tumors they produce, a limited angiogenic potential, and the frequent absence of histopathological features observed in corresponding patient tumors, such as necrosis [8].

\section{Patient-Derived Xenograft Models}

Human established cell lines (ECLs), continuously propagated as monolayer cultures in serum-supplemented media, such as the ubiquitous U87 MG line, have been used for establishing tumors in immunocompromised mice for more than 36 years [14], and some of the earliest established lines continue to be a staple of laboratories conducting preclinical therapeutic testing in rodents. An extensive review of glioma ECL tumorigenicity was published by Ishii et al. [15], and this work continues to serve as a valuable reference for investigators engaged in human glioma studies. Generally, xenografts established from ECLs are not referred to as patient-derived xenografts (PDX). The term, or the acronym, PDX is usually applied to tumors that are propagated in mice, rather than in cell culture. Admittedly, however, any xenograft established from human tumor cells, regardless of method of tumor cell propagation, is patient-derived.

With the intriguing potential and limited understanding of GEM model strengths and weaknesses at the outset of the GEM movement, interest in human tumor xenograft models waned during the rapid expansion period of GEM research. However, 2 high-impact studies prompted a resurgence of and have sustained a high level of interest in brain tumor xenograft models. The first was presented by Singh et al. in 2004 [16], and demonstrated the existence of human tumor cell subpopulations within individual patient surgical specimens, having distinct tumorigenic potential in immunocompromised mice. This landmark publication was followed by the study of Bao et al. [17], which showed differential sensitivity of human glioma cell subpopulations to radiation treatment. The 2 studies, in combination, stimulated and have maintained a high level of interest in 
research directed at understanding the dynamics of intratumoral subpopulation heterogeneity. Immunocompromised mice were, as well as continue to be, the tool with which to study subpopulation biologic characteristics of greatest interest: successful engraftment and engrafted tumor growth rate.

The decade 2000 to 2010 also proved to be a period during which there were substantial changes in approach to propagating human tumor tissues and cells. Highresolution molecular profiling studies have clearly established that sustained in vitro propagation of patient tumor explant cultures, with cells grown as monolayers in media supplemented with bovine sera, results in significant molecular and biologic changes to the tumor cells, in relation to the patient tumors from which they originated [18]. Studies that emerged and that showed improved retention of patient tumor characteristics through direct surgical specimen engraftment and propagation in immunocompromised mice [19], as well as by growth and propagation of surgical specimen explant cultures in media supplemented with specific amounts of defined growth factors that select for cancer stem cells [20], have had substantial influence on the ways in which patient tumors and cells are sustained for ongoing use in research.

In addition to the discovery of new approaches for propagating tumor tissue and cells, there has been increased attention directed to the type of immunodeficient mouse host used for tumor tissue engraftment and propagation. Successful transplantation of human tumor cells or tissue into mice requires neutralization and/or depletion of the adaptive immune response to avoid graft versus host immune-mediated tissue rejection. One of the most commonly utilized hosts for human tumor cell engraftment is the Foxn1-deficient nu/nu mouse strain, which is deficient for the thymus, a tissue required by lymphoid progenitor cells to undergo positive and negative selection that eventually produces naïve $\mathrm{T}$ cells and mature regulatory $\mathrm{T}$ cells [21]. The preferential use of nu/nu mice in cancer research is owing, in part, to historical rationale, as they were the first type of mouse to be widely available for human tumor xenograft establishment and propagation. Notably, they are relatively inexpensive, healthy (can survive as long as 2 years in an immunological barrier environment), and their lack of fur facilitates straightforward identification and quantification of tumors grown subcutaneously. Despite these attributes, athymic nu/nu mice likely introduce a bias for successful engraftment of surgical specimens, with successful engraftment mostly restricted to highly malignant variants within a histologic class of tumor. For brain tumors, this was indicated more than 27 years ago when it was shown that engrafted patient medulloblastomas frequently possess c-myc amplification [22]. Based on contemporary molecular classification, these tumors represent a subset of group 3 medulloblastomas [23], and are associated with highly malignant clinical behavior and poor prognosis for patients. Similarly, molecular profiling of GBM xenografts, established in athymic nu/nu mice, suggests a selection bias against the neuronal subgroup of these tumors [24].

Motivated by the need to expand tumor subtypes that can be successfully engrafted and propagated, mice with more severe immunodeficiency have experienced increasing use in xenograft-associated research. Examples include Rag1 or Rag2 knockout mice that are unable to form mature $\mathrm{T}$ and $\mathrm{B}$ cells, NOD-scid mice that are impaired for T- and B-cell lymphocyte development and are variably defective in natural killer cell function, and NOD-scid IL2rg ${ }^{\text {null }}$ mice that lack mature T and B cells, are natural killer cell deficient, and are variably defective in macrophage and dendritic cell function [25]. Potential barriers to working with severely immunodeficient mice is related to their high purchase price, their need for special care and housing, the increased incidence of host lymphoproliferative response to tissue engraftment that can mimic successful human tumor xenograft establishment [26], and the presence of fur which, to an extent, obscures subcutaneous tumor cell engraftment. Despite these challenges, the more severely immunocompromised status of such mice has helped to create new models, such as serially transplantable IDH1 mutant PDX [27, 28], established from lower-grade gliomas that do not engraft well, if at all, in athymic nu/nu mice.

Choosing the most appropriate mouse host for patient tumor engraftment is not only a vital consideration with respect to successful engraftment, but also with respect to testing therapies. Different strains of mice have inherent differences in chemotherapy and radiation sensitivity [29], which can be a limiting factor in the treatment regimen(s) that can be tested in conducting antitumor efficacy studies. Regardless of the type of immunocompromised mouse one chooses, any intention for large-scale engraftment-based research is well served by directing special attention to comparing the costs of purchasing from a vendor versus establishing and maintaining an in-house breeding colony, as the price for conducting large-scale PDX research can be cost-prohibitive.

Another important consideration for xenograft establishment and propagation concerns anatomic location: intracranial/orthotopic versus subcutaneous/heterotopic [30-32]. Whereas subcutaneous serial propagation of patient tumors has been demonstrated to maintain key molecular and biologic features of human brain tumors, as compared with propagation in vitro [19], the molecular and biologic characteristics of engrafted patient tumors diverge, to some extent, when propagating the same surgical specimen in heterotopic versus orthotopic location [33]. Orthotopic xenograft propagation has been 
confirmed to maximally retain corresponding patient tumor molecular characteristics [30, 33, 34]. However, a notable weakness of orthotopic transplantation is the uncertainty related to the length of time a mouse host can accommodate intracranial tumor before succumbing to tumor burden. Thus, orthotopically propagated tumors can be lost as a result of the unanticipated death of a tumor-bearing animal. Furthermore, orthotopic propagation is more limited with respect to the size of tumor a single animal can yield, which is an important consideration for experiments requiring a large number of cells from a single engrafted animal. Heterotopic propagation has practical advantages that include the ability to visualize directly tumor growth, avoiding unexpected tumorbearing animal deaths, and the generation of relatively large tumors that satisfy requirements for downstream experiments and further propagation.

A brain tumor PDX concept that has generated recent discussion involves the consideration of a PDX that can be generated and therapeutically tested within a time frame relevant for informing the treatment strategy of a patient from which the PDX is derived. This personalized approach, often referred to as "Avatar" modeling [35], is unrealistic in the vast majority of instances for brain tumor patients, given the latency period of initial PDX establishment, length of time required for PDX expansion, intracranial growth characterization, and subsequent therapeutic testing in vivo. A more realistic alternative involves the development of PDX panels that provide representation of several molecularly defined subclasses of a specific tumor histologic classification, such as GBM, and that could be used to test pre-existing and/or novel therapies. The results of testing such panels could then be used to select therapies, which are effective against a specific molecular subtype of PDX, in treating a corresponding patient whose tumor has a similar molecular profile as a responsive PDX [36, 37].

\section{Brain Tumor PDX Panel Opportunities}

As concerns the subject of tumor panels, it is notable that the vast majority of brain tumor ECL and PDX developed to date, and starting with the earliest examples [38, 39], are derived from patient surgical specimens classified as glioblastoma. However, and as described above, the increasing number of distinct types of immunocompromised mouse hosts are presenting opportunities for in vivo propagation of brain tumor histologies that have previously attracted little interest for use in preclinical studies to test therapeutic hypotheses. Below are a few examples for PDX panel opportunities that are based on our collective experience in establishing brain tumor xenografts.

\section{Primary Pediatric Brain Tumors}

Other than for medulloblastoma [40, 41], there has been surprisingly little presented in the literature that indicates attempts at establishing panels for specific types of pediatric brain tumors. Our recent efforts have focused on developing xenograft resources for therapeutic hypothesis testing in treating diffuse intrinsic pontine glioma, and specifically with inhibitors of histone post-translational modifiers [42, 43]. We have also developed xenograft models for testing inhibitors of BRAFV600E [44], which is a mutation observed in multiple types of brain tumor that occur predominantly in the pediatric population [45]. Our cumulative experience indicates that there are several additional pediatric brain tumor histologies that could be used in developing xenograft panels for specific types of pediatric brain tumors (Table 1 ).

\section{Meningiomas}

Meningiomas represent the most common primary brain tumor [46], but attract a level of preclinical research that is disproportionately low in comparison to their incidence. Whereas the basis for this disparity is multifactorial, there is little doubt of the primary basis for the modest level of preclinical research activity is owing to the majority of the tumors being benign and surgically curable. Nonetheless, significant fractions of all meningioma cannot be completely resected, and/or are of atypical or anaplastic classification, and these tumors generally recur, and are persistent disease, often and eventually leading to patient death. Similar to the situation with pediatric brain tumors there has been little effort directed towards developing meningioma cell line and/or xenograft panels [47], yet our experience indicates that establishing and sustaining human meningioma resources, as cell culture or as PDX, is very feasible (Table 1). Our analysis of meningioma PDX indicates that most surgical specimens that successfully engraft are both $N F 2$ /merlin- and CDKN2A (p16)deficient (Fig. 1), the latter of which has been noted in clinical specimens [49].

\section{Metastases to the Brain}

Metastases are the most common brain tumors and may occur up to 10 times more frequently than primary brain tumors in adults. They reportedly affect $9 \%$ to $17 \%$ of patients with cancer in large population- and autopsybased studies [50]. Our experience to date supports the feasibility of developing PDX from brain tumor metastases, which is perhaps not so surprising given that these tumors have already undergone malignant evolution in the patients from which they are obtained. Our 
Table 1 Cell lines and xenografts established from brain tumors other than adult glioblastoma multiforme (GBM)*, ${ }^{*}$

\begin{tabular}{llll}
\hline Tumor type & WHO grade & Cell line & Xenograft \\
\hline Pediatric brain tumors & & & \\
Astrocytoma & III & 3 & 0 \\
JPA & I & 8 & 0 \\
GBM & IV & 5 & 2 \\
DIPG & II & 1 & 1 \\
DIPG & III & 1 & 1 \\
DIPG & IV & 2 & 0 \\
Ependymoma & II & 3 & 1 \\
Ependymoma & III & 6 & 2 \\
Ependymoma & IV & 1 & 0 \\
Medulloblastoma & IV & 12 & 4 \\
AT/RT & IV & 4 & 2 \\
Meningiomas & & & \\
& I & 12 & 4 \\
II (atypical) & 12 & 4 \\
Brain tumor metastases & III (anaplastic) & 2 & 2 \\
Melanoma & & & 1 \\
Lung & & & 2 \\
Breast & & & 1 \\
Colon & & & \\
Kidney & & & \\
\hline
\end{tabular}

$\mathrm{WHO}=$ World Health Organization; JPA = juvenile pilocytic astrocyto$\mathrm{ma}$; DIPG = diffuse intrinsic pontine glioma; $\mathrm{AT} / \mathrm{RT}=$ atypical teratoid $/$ rhabdoid tumor

*All xenografts have been established in athymic nu/nu mice

${ }^{\dagger}$ Surgical specimens were minced with a scalpel and incubated in Papain solution to dissociate the tissue. The tissue was triturated using a sterile pipette until no clumps were visible, with the tissue suspension passed through a series of nylon mesh filters of 0.45 to $0.7 \mu \mathrm{m}$ pore diameter. After specimen centrifugation cell pellets were washed with phosphatebuffered saline and maintained as either a neurosphere culture or a monolayer culture in Dulbecco's modified eagle medium with $10 \%$ fetal bovine serum

comparison of patient brain tumor metastasis histology against derivative intracranial xenografts, following initial tumor establishment as a subcutaneous growth, show a remarkable preservation of patient tumor histology by the corresponding intracranial xenograft (Fig. 2). A complicating issue of developing PDX panels from tumors that have metastasized to the brain is the number of different organ sites from which primary cancer can spread to the brain (Table 1).

\section{Humanized Mice for Xenograft Research}

Brain tumor initiation and progression not only reflects the occurrence and accumulation of mutations, but also the

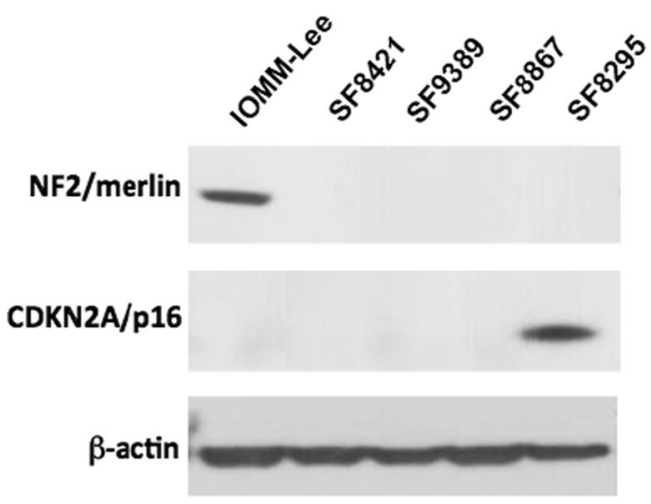

Fig. 1 Immunoblot results showing lack of expression of the $N F 2$ gene product, merlin, in 4 of 5 meningioma xenografts. The 1 positive xenograft was established from IOMM-Lee meningioma cells [48]. Four of the 5 xenografts are negative for expression of the CDKN2A gene product, p16. Surgical specimens from which SF8421 and SF8867 xenografts were established from tumors classified as anaplastic meningioma, whereas surgical specimens for SF9389 and SF8295 were established from atypical meningiomas

coincident failure of the immune system to control tumor growth. Understanding how tumors affect host immunity is therefore a critical topic of investigation for achieving increased understanding of cancer immunobiology and for identifying therapeutic strategies that engage patient immune response against their cancer. Much of our understanding of interrelationships between brain cancer and immune response has stemmed from the results of studies utilizing syngeneic mouse brain tumor models. However, substantial differences exist between murine and human immune function, as well as cancer biology, so extrapolating from mouse to human may often carry with it a number of erroneous assumptions. The use of PDX models has largely precluded the study of immune response to tumor, owing to the immunocompromised status of host mice. Recently, however, a humanized mouse model has been described whereby NOD-scid IL $2 r^{\text {null }}$ mice are engrafted with human fetal thymus and fetal liver-derived hematopoietic stem cells $[51,52]$. Engrafted mice develop a mature immune system that includes human myeloid cells, $\mathrm{T}$ cells, and B cells. Through human leukocyte antigenmatching one can investigate human-on-human immune system-tumor interactions. Reports of humanized mouse models for studying human cancer are thus far infrequent, but seem likely to see substantial increase given the high level of interest in studying immunotherapies for treating cancer.

\section{Approaches for Monitoring Intracranial Tumor Growth and Response the Therapy}

Survival analysis is the gold standard for conducting therapy-response studies with rodents bearing intracranial 
Fig. 2 Histologic comparisons between original brain metastases and corresponding patientderived xenograft from athymic $\mathrm{nu} / \mathrm{nu}$ mouse brain, and following initial subcutaneous. NU035, which was developed from a metastatic mucinous micropapillary adenocarcinoma of the lung, retains the original micropapillary architecture and still produces mucin. Scale bar $=$ $50 \mu \mathrm{m}$

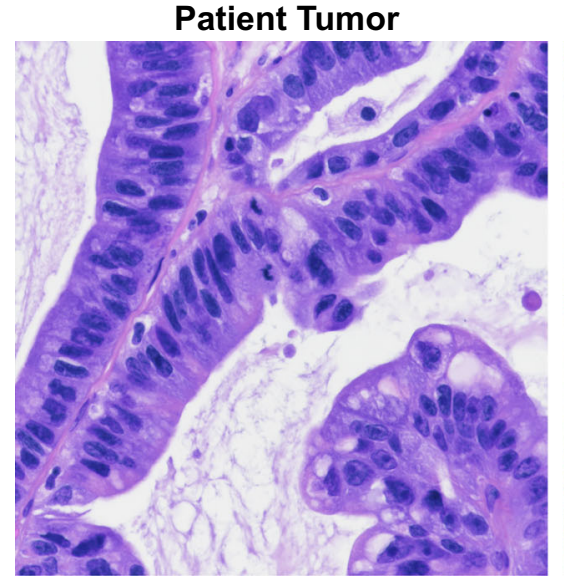

Derivative Intracranial Xenograft

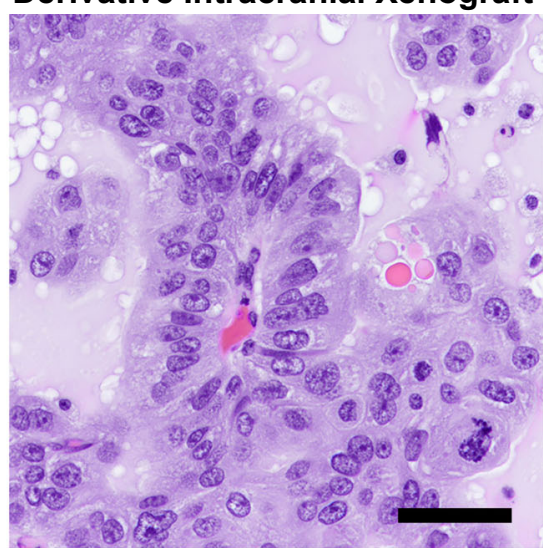

tumors, whether engrafted, induced, or spontaneously occurring. However, survival provides a single metric from what is often a costly, and lengthy, experiment. Commonly used methods for obtaining in-experiment feedback, to complement survival results, include the timed euthanasia of animal subjects while on therapy, with subsequent analysis of brain tumor cell indicators of therapeutic activity, such as Ki-67 antibody staining for addressing proliferation effects of treatment, and terminal deoxynucleotidyl transferase dUTP nick end labeling staining for determination of treatment effects on cell death. In immunocompetent animals undergoing immunotherapeutic evaluation, defined time point analyses are often used to analyze animal subjects for circulating immune cells and/or for immune cell infiltrates in intracranial tumor.

Tumor imaging methods, for obtaining in-experiment results on intracranial tumor response to treatment, have seen steadily increasing use in recent years. Longitudinal tumor imaging of live animal subjects include magnetic resonance imaging (MRI), fluorescent optical imaging, and positron emission tomography [53]. Additionally, bioluminescence imaging (BLI) is frequently used to detect the emission of photons from energy-dependent reactions involving the metabolism of exogenous luciferin substrate by tumor cells that have been genetically modified to express luciferase. BLI studies have demonstrated a strong correlation between tumor volume and treatment response [54], similar to MRI, with the benefit of a lower cost to operate, as well as lower overall labor requirement [55]. Furthermore, the use of gadolinium-enhanced MRI normally requires the presence of specialized personnel for technical operation, and is often limited to analyzing one animal subject at a time, which results in a severalfold increase in imaging time for a series of mice [55]. Also, and unlike fluorescent imaging of labeled tumor cells for which signal-to-noise ratios are problematic owing to high normal tissue autofluorescence, photon scattering, and fluorophore photo-bleaching, BLI possesses minimal background activity, facilitating a remarkably sensitive quantification of increasing, or decreasing, tumor size $[54,55]$. The use of synthetic luciferin analogues, such as Cycluc1 [56] and AkaLumine-HCl [57], is increasing in xenograft BLI studies owing to superior biodistribution and energy emission properties, relative to luciferin, that heighten the sensitivity of luciferasemodified tumor cell detection. Regardless of the approach utilized, the incorporation of a tumor-imaging method is an important consideration for obtaining in-experiment feedback regarding therapeutic activity, or lack thereof.

\section{Conclusions}

Preclinical investigation of novel therapeutics and administration strategies that utilize rodent brain tumor models are likely to remain an essential part of preclinical research for translation of bench-to-bedside laboratory-based discoveries. As reviewed above, a number of options are available for facilitating and promoting discovery leading to improved care and outcomes for patients with a brain tumor. Rodent models are tools to be used for enabling discovery, and, as is the case for any tool, it is important that the "tradesman" knows which tools are most appropriate for a given circumstance. In this review we have provided an overview of available rodent models, or tools, and we look forward to reading of future discoveries from their application.

Acknowledgements D.A.W. is supported by Public Health Service (PHS) grant number R00NS082381 and R01NS097851. R.H. is supported by PHS grant number R01NS093079. C.D.J. is supported by PHS grant numbers R01CA159467, R01NS080619, and R01NS095642.

Required Author Forms Disclosure forms provided by the authors are available with the online version of this article.

Publisher's Note Springer Nature remains neutral with regard to jurisdictional claims in published maps and institutional affiliations. 


\section{References}

1. Donehower LA, Harvey M, Slagle BL, et al. Mice deficient for $\mathrm{p} 53$ are developmentally normal but susceptible to spontaneous tumours. Nature 1992;356:215-221.

2. Ding H, Roncari L, Shannon P, et al. Astrocyte-specific expression of activated p21-ras results in malignant astrocytoma formation in a transgenic mouse model of human gliomas. Cancer Res 2001;61: 3826-3836.

3. Gutmann DH, Stiles CD, Lowe SW, Bollag GE, Furnari FB, Charest AL. Report from the fifth National Cancer Institute Mouse Models of Human Cancers Consortium Nervous System Tumors Workshop. Neuro Oncol 2011;13:692-699.

4. Zhu Y, Guignard F, Zhao D, et al. Early inactivation of p53 tumor suppressor gene cooperating with NF1 loss induces malignant astrocytoma. Cancer Cell 2005;8:119-130.

5. Cancer Genome Atlas Research Network. Comprehensive genomic characterization defines human glioblastoma genes and core pathways. Nature 2008;455:1061-1068.

6. Kwon CH, Zhao D, Chen J, et al. PTEN haploinsufficiency accelerates formation of high-grade astrocytomas. Cancer Res 2008;68: 3286-3294.

7. Chen L, Zhang Y, Yang J, Hagan JP, Li M. Vertebrate animal models of glioma: understanding the mechanisms and developing new therapies. Biochim Biophys Acta 2013;1836:158-165.

8. Becher OJ, Holland EC. Genetically engineered models have advantages over xenografts for preclinical studies. Cancer Res 2006;66:3355-3358.

9. Barth RF, Kaur B. Rat brain tumor models in experimental neurooncology: the C6, 9L, T9, RG2, F98, BT4C, RT-2 and CNS-1 gliomas. J Neurooncol 2009;94:299-312.

10. Oh T, Fakurnejad S, Sayegh ET, et al. Immunocompetent murine models for the study of glioblastoma immunotherapy. J Transl Med 2014;12:107

11. Wainwright DA, Chang AL, Dey M, et al. Durable therapeutic efficacy utilizing combinatorial blockade against IDO, CTLA-4 and PD-L1 in mice with brain tumors. Clin Cancer Res 2014; 20 : 5290-5301

12. Belcaid Z, Phallen JA, Zeng J, et al. Focal radiation therapy combined with 4-1BB activation and CTLA-4 blockade yields longterm survival and a protective antigen-specific memory response in a murine glioma model. PLOS ONE 2014;9:e101764.

13. Fecci PE, Ochiai H, Mitchell DA, et al. Systemic CTLA-4 blockade ameliorates glioma-induced changes to the $\mathrm{CD} 4+\mathrm{T}$ cell compartment without affecting regulatory T-cell function. Clin Cancer Res 2007;13:2158-2167.

14. Bigner SH, Bullard DE, Pegram CN, Wikstrand CJ, Bigner DD. Relationship of in vitro morphologic and growth characteristics of established human glioma-derived cell lines to their tumorigenicity in athymic nude mice. J Neuropathol Exp Neurol 1981;40:390-409.

15. Ishii N, Maier D, Merlo A, et al. Frequent co-alterations of TP53, p16/CDKN2A, p14ARF, PTEN tumor suppressor genes in human glioma cell lines. Brain Pathol 1999;9:469-479.

16. Singh SK, Hawkins C, Clarke ID, et al. Identification of human brain tumour initiating cells. Nature 204;432:396-401

17. Bao $\mathrm{S}, \mathrm{Wu} \mathrm{Q}, \mathrm{McLendon} \mathrm{RE}$, et al. Glioma stem cells promote radioresistance by preferential activation of the DNA damage response. Nature 2006;444:756-760.

18. Li A, Walling J, Kotliarov Y, et al. Genomic changes and gene expression profiles reveal that established glioma cell lines are poorly representative of primary human gliomas. Mol Cancer Res 2008;6: 21-30.

19. Giannini C, Sarkaria JN, Saito A, et al. Patient tumor EGFR and PDGFRA gene amplifications retained in an invasive intracranial xenograft model of glioblastoma multiforme. Neuro Oncol 2005;7: 164-176

20. Pollard SM, Yoshikawa K, Clarke ID, et al. Glioma stem cell lines expanded in adherent culture have tumor-specific phenotypes and are suitable for chemical and genetic screens. Cell Stem Cell 2009;4:568-580.

21. Kelland LR. Of mice and men: values and liabilities of the athymic nude mouse model in anticancer drug development. Eur J Cancer 2004;40:827-836

22. Bigner SH, Friedman HS, Vogelstein B, Oakes WJ, Bigner DD. Amplification of the c-myc gene in human medulloblastoma cell lines and xenografts. Cancer Res 1990;50:2347-2350

23. Massimino M, Biassoni V, Gandola L, et al. Childhood medulloblastoma. Critical Rev Oncol Hematol 2016;105:35-51.

24. Verhaak RG, Hoadley KA, Purdom E, et al. Integrated genomic analysis identifies clinically relevant subtypes of glioblastoma characterized by abnormalities in PDGFRA, IDH1, EGFR, and NF1. Cancer Cell 2010;17:98-110.

25. Shultz LD, Goodwin N, Ishikawa F, Hosur V, Lyons BL, Greiner DL. Human cancer growth and therapy in immunodeficient mouse models. Cold Spring Harb Protoc 2014;2014:694-708.

26. Bondarenko G, Ugolkov A, Rohan S, et al. Patient-derived tumor xenografts are susceptible to formation of human lymphocytic tumors. Neoplasia 2015;17:735-41.

27. Klink B, Miletic H, Stieber D, et al. A novel, diffusely infiltrative xenograft model of human anaplastic oligodendroglioma with mutations in FUBP1, CIC, and IDH1. PLOS One 2013;8:e59773.

28. Luchman HA, Stechishin OD, Dang NH, et al. An in vivo patientderived model of endogenous IDH1-mutant glioma. Neuro Oncol 2012;14:184-191.

29. Nowosielska EM, Cheda A, Wrembel-Wargocka J, Janiak MK. Effect of low doses of low-let radiation on the innate anti-tumor reactions in radioresistant and radiosensitive mice. Dose Resp 2012;10:500-515

30. Joo KM, Kim J, Jin J, et al. Patient-specific orthotopic glioblastoma xenograft models recapitulate the histopathology and biology of human glioblastomas in situ. Cell Rep 2013;3:260-273.

31. Carlson BL, Pokorny JL, Schroeder MA, Sarkaria JN. Establishment, maintenance and in vitro and in vivo applications of primary human glioblastoma multiforme (GBM) xenograft models for translational biology studies and drug discovery. Curr Protoc Pharmacol 2011; Chapter 14:Unit 14. 16.

32. Hodgson JG, Yeh R-F, Ray A, et al. Comparative analyses of gene copy number and mRNA expression in glioblastoma multiforme tumors and xenografts. Neuro Oncol 2009;11:477-487.

33. Atunes L, Angioi-Duprez KS, Bracard SR, et al. Analysis of tissue chimerism in nude mouse brain and abdominal xenograft models of human glioblastoma multiforme: what does it tell us about the models and about glioblastoma biology and therapy? J Histochem Cytochem 2000;48:847-858.

34. Wang J, Miletic H, Sakariassen PO, et al. A reproducible brain tumour model established from human glioblastoma biopsies. BMC Cancer 2009;9:465.

35. Garralda E, Paz K, Lopez-Casas PP, et al. Integrated nextgeneration sequencing and avatar mouse models for personalized cancer treatment. Clin Cancer Res 2014;20:2476-2484.

36. Behrens D, Rolff J, Hoffmann J. Predictive in vivo models for oncology. Handb Exp Pharmacol 2016;232:203-221.

37. Burgenske DM, Monsma DJ, Dylewski D, et al. Establishment of genetically diverse patient-derived xenografts of colorectal cancer. Am J Cancer Res 2014;4:824-837.

38. Mark J, Ponten J, Westermark B. Cytogentical studies with G-band technique of established cell lines of human malignant glomas. Hereditas 1974;78:304-307

39. Sarkaria JN, Carlson BL, Schroeder MA, et al. Use of an orthotopic xenograft model for assessing the effect of epidermal growth factor 
receptor amplification on glioblastoma radiation response. Clin Cancer Res 2006;12:2264-2271.

40. He XM, Skapek SX, Wikstrand CJ, et al. Phenotypic analysis of four human medulloblastoma cell lines and transplantable xenografts. J Neuropathol Exp Neurol 1989;48:48-68.

41. Sterling-Levis K, White L. The role of xenografting in pediatric brain tumor research with specific emphasis on medulloblastoma/primitive neuroectodermal tumors of childhood. In Vivo 2003;17:329-342.

42. Hashizume R, Andor N, Ihara Y, et al. Pharmacologic inhibition of histone demethylation as a therapy for pediatric brainstem glioma. Nat Med 2014;20:1394-1396.

43. Piunti A, Hashizume R, Morgan MA, et al. Heterotypic nucleosomes and PRC2 drive DIPG oncogenesis. Nat Med 2017 Feb 27.

44. Nicolaides TP, Li H, Solomon DA, et al. Targeted therapy for BRAFV600E malignant astrocytoma. Clin Cancer Res 2011;17: 7595-7604.

45. Schindler G, Capper D, Meyer J, et al. Analysis of BRAF V600E mutation in 1,320 nervous system tumors reveals high mutation frequencies in pleomorphic xanthoastrocytoma, ganglioglioma and extra-cerebellar pilocytic astrocytoma. Acta Neuropathol 2011;121:397-405.

46. Fathi AR, Roelcke U. Meningioma. Curr Neurol Neurosci Rep 2013;13:337.

47. Ragel BT, Couldwell WT, Gillespie DL, Wendland MM, Whang K, Jensen RL. A comparison of the cell lines used in meningioma research. Surg Neurol 2008;70:295-307.

48. Lee WH. Characterization of a newly established malignant meningioma cell line of the human brain: IOMM-Lee. Neurosurgery 1990;27:389-395.

49. Boström J, Meyer-Puttlitz B, Wolter M, et al. Alterations of the tumor suppressor genes CDKN2A (p16(INK4a)), p14(ARF),
CDKN2B (p15(INK4b)), and CDKN2C (p18(INK4c)) in atypical and anaplastic meningiomas. Am J Pathol 2001;159:661-669.

50. Takei H, Rouah E, Ishida Y. Brain metastasis: clinical characteristics, pathological findings and molecular subtyping for therapeutic implications. Brain Tumor Pathol 2016;33:1-12.

51. Aryee KE, Shultz LD, Brehm MA. Immunodeficient mouse model for human hematopoietic stem cell engraftment and immune system development. Methods Mol Biol 2014;1185:267-278.

52. Hasgur S, Aryee KE, Shultz LD, Greiner DL, Brehm MA. Generation of immunodeficient mice bearing human immune systems by the engraftment of hematopoietic stem cells. Methods Mol Biol 2016;1438: 67-78.

53. Wang Y, Tseng JC, Sun Y, Beck AH, Kung AL. Noninvasive imaging of tumor burden and molecular pathways in mouse models of cancer. Cold Spring Harb Protoc 2015;2015:135-144.

54. Dinca EB, Sarkaria JN, Schroeder MA, et al. Bioluminescence monitoring of intracranial glioblastoma xenograft: response to primary and salvage temozolomide therapy. J Neurosurg 2007;107:610-616.

55. Szentirmai O, Baker $\mathrm{CH}$, Lin $\mathrm{N}$, et al. Noninvasive bioluminescence imaging of luciferase expressing intracranial U87 xenografts: correlation with magnetic resonance imaging determined tumor volume and longitudinal use in assessing tumor growth and antiangiogenic treatment effect. Neurosurgery 2006;58:365-372.

56. Evans MS, Chaurette JP, Adams ST Jr, et al. A synthetic luciferin improves bioluminescence imaging in live mice. Nat Methods 2014;11:393-395.

57. Kuchimaru T, Iwano S, Kiyama M, et al. A luciferin analogue generating near-infrared bioluminescence achieves highly sensitive deep-tissue imaging. Nat Commun 2016;7:11856. 